Espacio y Desarrollo № 27, 2015, pp. 137-158 (ISSN 1016-9148)

http://dx.doi.org/10.18800/espacioydesarrollo.201501.007

\title{
Los territorios del agua en dos localidades de la Amazonía NORTe del Perú: los Casos de Lamas (San Martín) y Santa María de Nieva (Amazonas)
}

\author{
Gustavo Rondón Ramírez \\ Universtité Catholique de Louvain (URBA) \\ Pontificia Universidad Católica del Perú, \\ Centro de Investigación de la Arquitectura y la Ciudad (CIAC) \\ grondon@pucp.edu.pe
}

\section{Resumen}

En los últimos 50 años, el crecimiento de la población que vive en zonas urbanas se ha incrementado de manera notable en Latinoamérica. Si bien este crecimiento se concentra principalmente en las ciudades costeras, espacios antes menos poblados e incluso percibidos erróneamente como deshabitados, como la Amazonía, también presentan un incremento poblacional urbano. Las ciudades de Lamas y Santa María de Nieva pertenecen a la red de ciudades del norte del Perú: están situadas en un eje transversal que conecta la costa (Piura, Chiclayo) con la baja Amazonía (Iquitos). Teniendo en cuenta las características de ocupación del territorio y a través de un levantamiento de información de campo realizado durante el mes de agosto del 2015, sumado a la utilización de fuentes cartográficas (mapas e imágenes satelitales) se realizó un primer análisis del ciclo urbano del agua, basado en la idea de que este ciclo no solo se realiza de manera íntegra en el ámbito urbano y que evidentemente presenta una relación estrecha con el territorio (y sus recursos) que rodea a ambas localidades. Los resultados obtenidos muestran que a nivel fuentes de agua y retorno de las aguas utilizadas, ambas localidades tienen un gran impacto (negativo y positivo) en el territorio; además, se pudo identificar que dentro del espacio urbano el agua también es un elemento que configura o delimita subzonas.

Palabras clave: territorios del agua, Lamas, Santa María de Nieva, ciclo urbano del agua.

The waterscapes in two touns in the northern Amazonia of Peru: the cases of Lamas (San Martín) and Santa María de Nieva (Amazonas)

\section{Abstract}

In the last 50 years, the population growth of urban areas has increased significantly in Latin America. While this growth is mainly concentrated in coastal cities, places that were less populated before and even misperceived as uninhabited, as the Amazonia, also present an urban population increase. The cities of Lamas and Santa María de Nieva belong to the network 
of Peruvian northern cities: they are located in a transverse axis connecting the coast (Piura, Chiclayo) with the lower Amazonia (Iquitos). Taking into account the characteristics of land occupation, using cartographic sources (maps and satellite images) and gathering data from field research conducted during the month of August 2015, a first analysis of the urban water cycle was performed. It is based on the idea that this cycle is not only carry out in urban areas but rather has a close relationship with the territory (and resources) surrounding both locations. The results show that both cities have a significant impact (negative and positive) in the water sources and wastewater discharges of the territory. It has also been found that inside the urban space, water is an element that configures or sets sub-zones.

Keywords: Waterscapes, Lamas, Santa María de Nieva, urban water cycle

\section{INTRODUCCIÓN}

En los últimos 50 años, el crecimiento de la población que vive en zonas urbanas se ha incrementado de manera notable en Latinoamérica. Si bien este crecimiento se concentra principalmente en las ciudades costeras, espacios antes menos poblados e incluso percibidos erróneamente como deshabitados, como es el caso de la Amazonía, también presentan un incremento de la población urbana. Los grandes ríos ya no son las únicas vías para la entrada-salida de productos comerciales, para el transporte de personas y no son los únicos elementos que atraen el asentamiento de la población. Con la construcción y mejoramiento de las carreteras se ha facilitado el proceso de ocupación del espacio amazónico porque se permite un intercambio fluido de bienes pero también de ideas y costumbres (tanto de la costa como de la sierra de nuestro país). Esta nueva dinámica territorial también tiene impacto en la forma cómo se utilizan los recursos del territorio y en la forma cómo las sociedades se relacionan con los recursos naturales, especialmente el agua.

En ninguna parte del mundo el agua se considera solo como un recurso natural, todas las culturas del mundo le han asignado valores siempre trascendentales (Cabrera, 2014). Tanto en culturales occidentales como orientales, andinas o amazónicas, el agua ha sido más que un elemento del territorio, muchas veces ha sido vista como divinidad, fuente de vida y origen de civilizaciones. Por lo tanto, es una divinidad que está presente en los lagos, las lagunas, el mar, los ríos y todas las fuentes de agua (Agurto Belloso, 2006). El agua es también base de la reciprocidad y complementariedad porque permite la integración de los seres vivos, la articulación de la naturaleza y de la sociedad humana; que permite la participación de la población, permitiendo la autodeterminación de las comunidades y dialogo permanente con la naturaleza (Cabrera, 2014).

Sin embargo, a raíz del crecimiento de la población urbana en zonas donde antes la ocupación era dispersa y del nuevo modelo económico-productivo de explotación de recursos naturales, el agua se ha convertido, predominantemente, en un factor de producción (Aguilera, 2006). Por esa razón, autores como Swyngedouw (2004, 2009); 
Linton (2010); Budds (2012) señalan que se debe analizar la relación entre la sociedad y el agua como un solo conjunto, un ciclo hidrosocial que representa a todo nivel la relación de esos dos elementos en un mismo territorio.

\section{Contexto amazónico: agua, territorio y sociedad}

La realidad tan particular y compleja planteada en la introducción, es la base para estudiar las relaciones entre la utilización de los recursos naturales (agua y suelo) en dos espacios urbanos de la Amazonía norte del Perú. Si bien es innegable que la Amazonía es por excelencia el territorio del agua del Perú, hoy en día las nuevas dinámicas territoriales, antes mencionadas, nos invitan a reflexionar sobre cómo la presencia de centros urbanos y actividades económicas-productivas cambian esta percepción de la abundancia real de agua y configuran nuevos territorios donde se da la paradoja en que este recurso agua se ha convertido en un bien escaso.

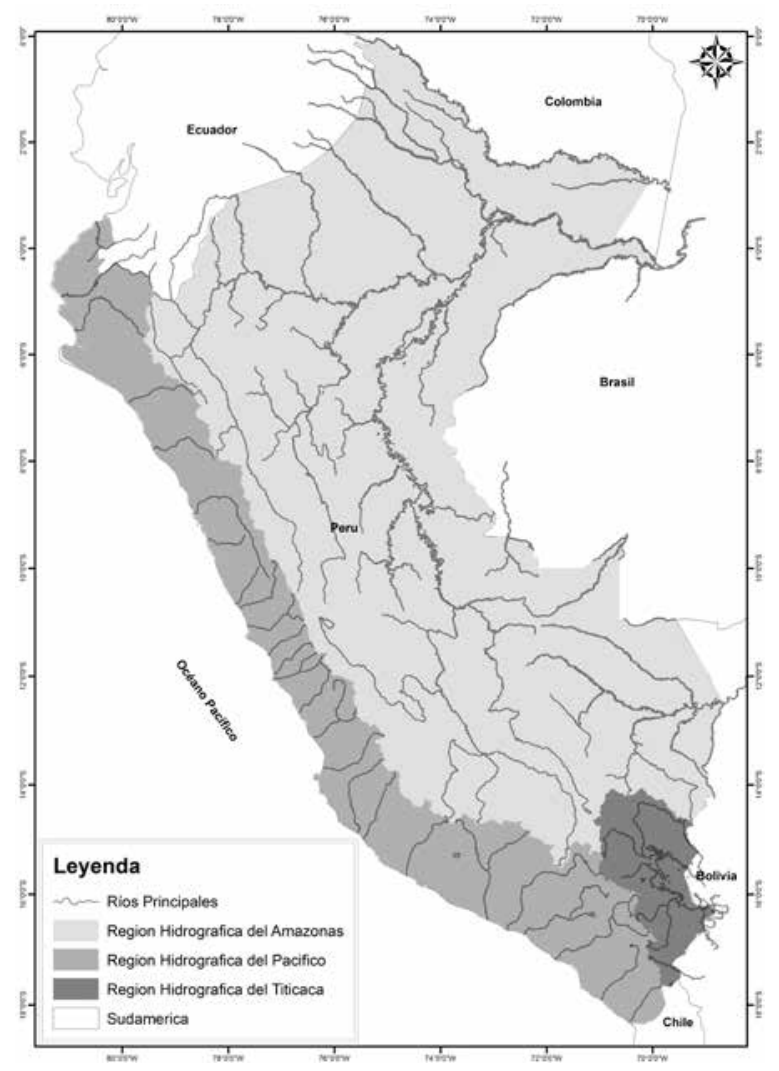

Figura 1. Mapa de las vertientes hidrográficas en el Perú.

Fuente: ESRI Images, Ministerio de Transportes y Comunicaciones (MTC),

Instituto Geográfico Nacional (IGN). Elaboración: Gustavo Rondón R. 
En primer lugar, es común leer o escuchar que el Perú presenta una asimetría en la disponibilidad del recurso hídrico (De la Torre, 2012). El 97,27\% de la disponibilidad física del agua se encuentra en la cuenca del Amazonas (donde vive el 30\% de la población nacional), el 2,18\% en la vertiente del Pacífico (donde vive el 66\% de la población nacional) y solo el $0,56 \%$ se encuentra en la cuenca del lago Titicaca (donde vive en el $4 \%$ de la población nacional).

En los últimos años, debido al crecimiento de la población del país, la rápida urbanización y el modelo de desarrollo económico basado en la exportación de materias primas, la calidad del agua se ha deteriorado rápidamente. La contaminación de los ríos, lagos y mares merece una atención especial debido a su impacto negativo. Los vertimientos de residuos sólidos y aguas residuales generadas por la población, la minería informal, los pasivos mineros, la industria, la contaminación difusa por el uso de productos químicos agrícolas, la explotación de hidrocarburos y la industria extractiva de madera son las principales actividades que generan un impacto negativo en la calidad del agua (De la Torre, 2012).

Finalmente, en la Figura 2, se puede observar que, además de los problemas de agua, en la Amazonía hay una superposición de concesiones madereras, concesiones mineras y las concesiones petroleras con las áreas de las comunidades nativas, reservas territoriales y zonas naturales protegidas. Este contexto de superposición permite el desarrollo de conflictos sociales estrechamente vinculados a la gestión del agua (cantidad y calidad) y los derechos de uso de la tierra. Según Pereyra (2005), de estos conflictos, que se han triplicado en número y en nivel de violencia, el $26 \%$ de los conflictos están relacionados con cuestiones agrícolas (cantidad de agua) y $80 \%$ de los conflictos están relacionados con la calidad del agua. Sin embargo, de acuerdo con la Defensoría del Pueblo, en su informe correspondiente al mes de mayo de 2015, en el Perú existen 152 conflictos socioambientales activos donde el 66,4\% (95 casos) corresponden a actividades relacionadas con la minería; seguido de los conflictos generados por la actividad petrolera con el 14,7\% (21 casos) En todos los casos, es una preocupación para la población local de los impactos que las actividades económicas pueden tener en la cantidad y la calidad de sus aguas.

Entonces la pregunta que surge sobre el contexto territorial donde se desarrolla la investigación es muy puntual: ¿Cómo definimos a la Amazonía? En el Perú existen diversas definiciones de la Amazonía según el o los criterios adoptados. El más común está relacionado con el bosque. La «selva» peruana ha sido dividida en dos por el geógrafo peruano Javier Pulgar-Vidal $(1982,2014)$ en su libro Las 8 regiones naturales del Perú, donde el autor hace una distinción entre la «selva alta» y la "selva baja». Sin embargo, Pulgar-Vidal no ha sido el único que a lo largo de los años ha tratado de definir a la «selva» del Perú. Distintos investigadores, basados en su propia formación y campo de estudio, han tratado de definir esta región del Perú. Bernex y Montes (1992), presentan una 


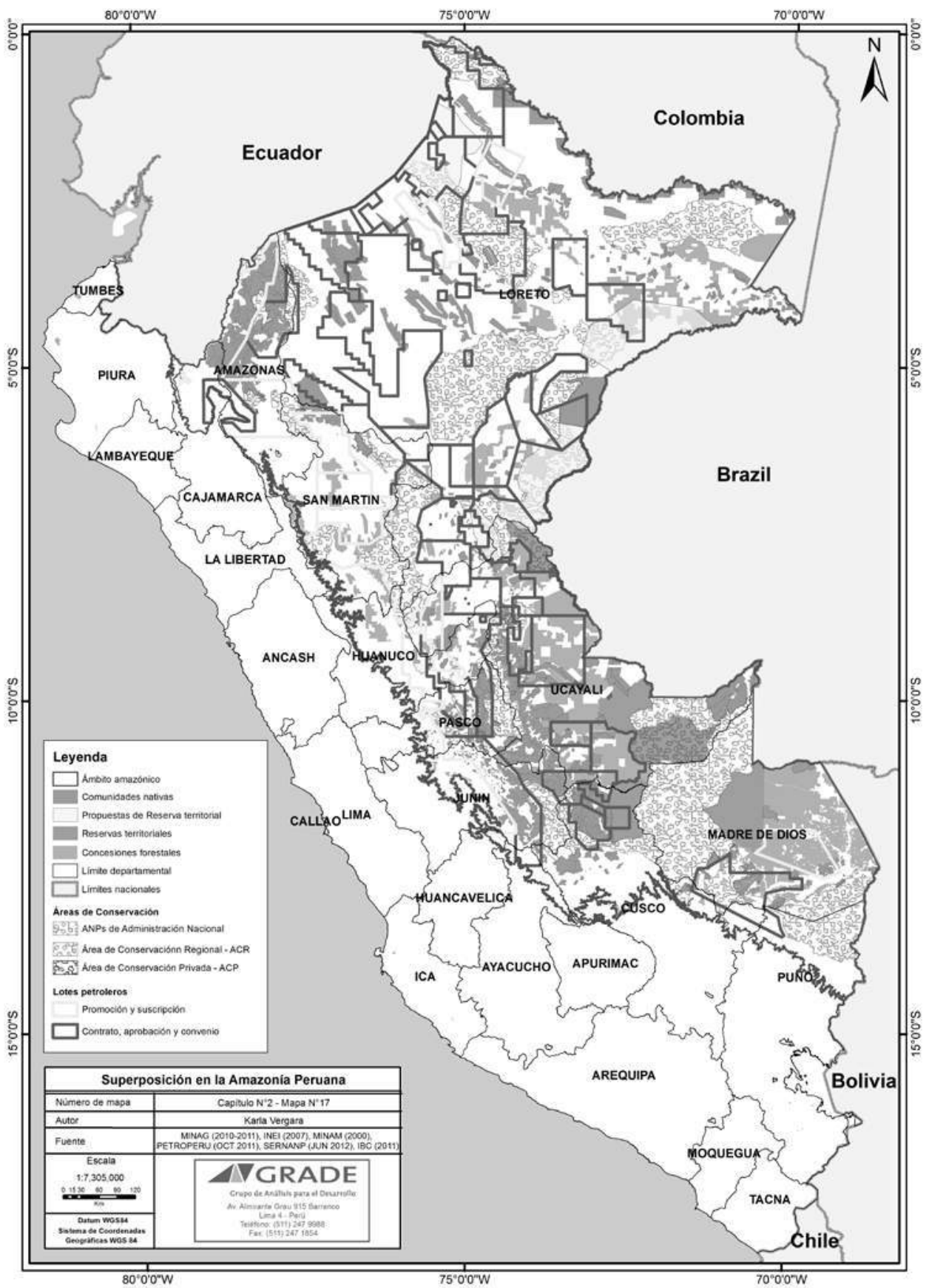

Figura 2. Mapa de superposición de derechos de suelo en la Amazonía del Perú. Fuente: GRADE-IEP, 2014. Elaboración: K. Vergara. 
recopilación de los esfuerzos más importantes. Así, según Petersen, la Amazonía se puede dividir en una primera zona con más de 2500 metros de altitud, con precipitaciones entre $600 \mathrm{~mm}$ y $1000 \mathrm{~mm}$ por año; una segunda zona situada entre los 500 y 1500 metros de altitud, con precipitaciones entre 1000 y $2000 \mathrm{~mm}$ por ańo; y una tercera zona, a menos de 500 metros de altitud, con características tropicales, con precipitaciones de $2000 \mathrm{~mm}$ a $3000 \mathrm{~mm}$ por año. Como podemos observar, esta clasificación se basa en dos factores: la altitud y la cantidad de precipitación registrada. Otros autores, como Peñaherrera, han utilizado para la clasificación la variable relieve o la vegetación que fue el criterio seleccionado por Weberbauer o los recursos forestales, variable que utilizó Mailleux. Esta variedad de criterios muestra la complejidad en lograr una definición de la Amazonía (haciendo énfasis en la dificultad de definir la alta Amazonía).

Las definiciones creadas por Pulgar-Vidal y otros autores en la segunda mitad del siglo XX se basan en aspectos topográficos, hidrográficos y ecológicos. Hoy en día, las dinámicas territoriales han transformado la Amazonía del Perú muy rápidamente y a veces, peligrosamente. Por ejemplo, podemos distinguir entre la Amazonía de los grandes ríos (las vías naturales de comunicación de esta región, que con la explotación de los recursos naturales — caucho, madera, etc.— se han convertido en las rotas de penetración y salida de los productos comerciales); la Amazonía de las carreteras (que facilitan la ocupación del espacio amazónico); la Amazonía de las ciudades como Iquitos, Pucallpa o Tarapoto con dinámicas comerciales muy fuertes; la Amazonía de las comunidades nativas con una débil ocupación del territorio, una gran dispersión y una manera diferente de utilizar los recursos naturales. Esto nos lleva a decir que tal vez sería apropiado empezar a comprender que no existe «una» Amazonía, sino que estamos ante «múltiples» amazonías.

A nivel político-administrativo la Amazonía tiene tres niveles de la demarcación, basados en la división político-administrativa vigente en el Perú: el nivel regional, provincial y distrital. A estos tres niveles de gobierno hay que añadir, transversalmente, el poder ejercido por los distintos sectores a nivel estatal, siendo los ministerios responsables de tomar decisiones relacionadas con el territorio. Muchas políticas de Estado propuesta por el Ministerio de Energía y Minas pueden superponerse y oponerse con las del Ministerio de Agricultura y Riego, o viceversa, incluida las políticas que nacen desde la Autoridad Nacional del Agua.

Basado en todo lo seńalado anteriormente y reconociendo la importancia de las características físico-ambientales y socioculturales, podemos elegir definiciones más territoriales para la Amazonía, como la Amazonía de las carreteras (alta Amazonía); la Amazonía de los grandes ríos (Amazonía baja); la Amazonía de las ciudades, la Amazonía de las comunidades nativas, la Amazonía las cuencas hidrográficas y la Amazonía institucional. Es este el contexto territorial es que se encuentran ubicadas nuestras dos ciudades de estudio. 


\section{El contexto territorial de las localidades de estudio}

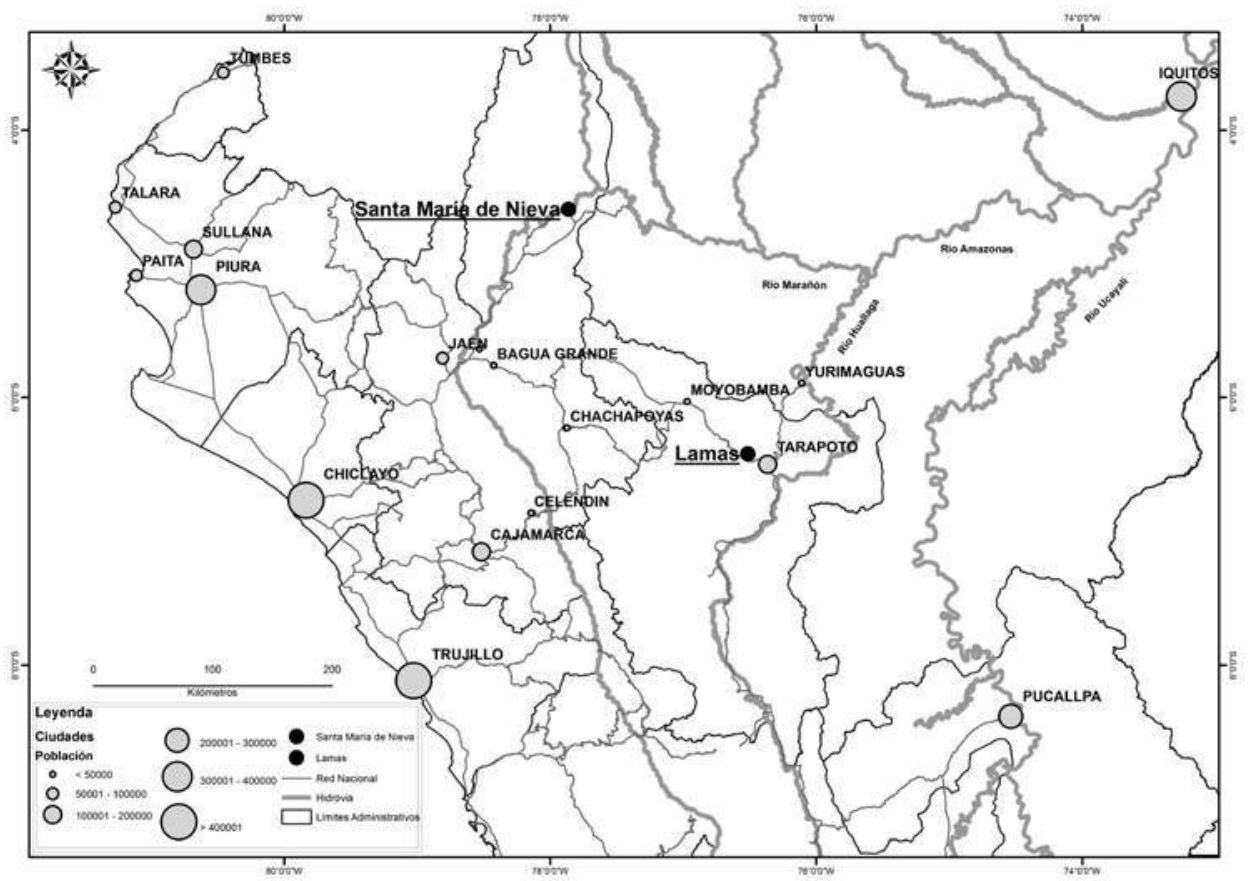

Figura 3. Las ciudades de estudio en el contexto de la región norte del Perú. Fuentes: ESRI Images, Ministerio de Transportes y Comunicaciones (MTC). Elaboración: Gustavo Rondón R.

Tanto Lamas como Santa María de Nieva pertenecen a la red de ciudades del norte de Perú: están situadas en un eje transversal que conecta la costa (Piura, Chiclayo) con la baja Amazonía (Iquitos).

Si bien el contexto geográfico en que se encuentran ambas ciudades son distintos (Lamas está ubicada en una zona de relieve montańoso y Nieva en la confluencia de los ríos Marańón y Nieva), ambas son capitales provinciales por lo que cumplen una función administrativa clave para la población que vive en sus hinterlands y ambas presentan una población indígena-nativa que ha ocupado esos territorios mucho antes de la consolidación de estos espacios urbanos.

La ciudad de Lamas se encuentra ubicada en la región San Martín, provincia de Lamas y distrito de Lamas. Tiene una población de 8883 habitantes (INEI, 2007) y como capital provincial concentra los poderes administrativos. Si tenemos en cuenta que la población total del distrito es 13173 habitantes (donde el 82\% es considerada población urbana), Lamas concentra a más de la mitad (68\% aprox.) de la población urbana del distrito. Las actividades económicas principales son el comercio, los servicios y la agroforestería (principalmente los cultivos de café y cacao, desarrollados en su hinterland). 


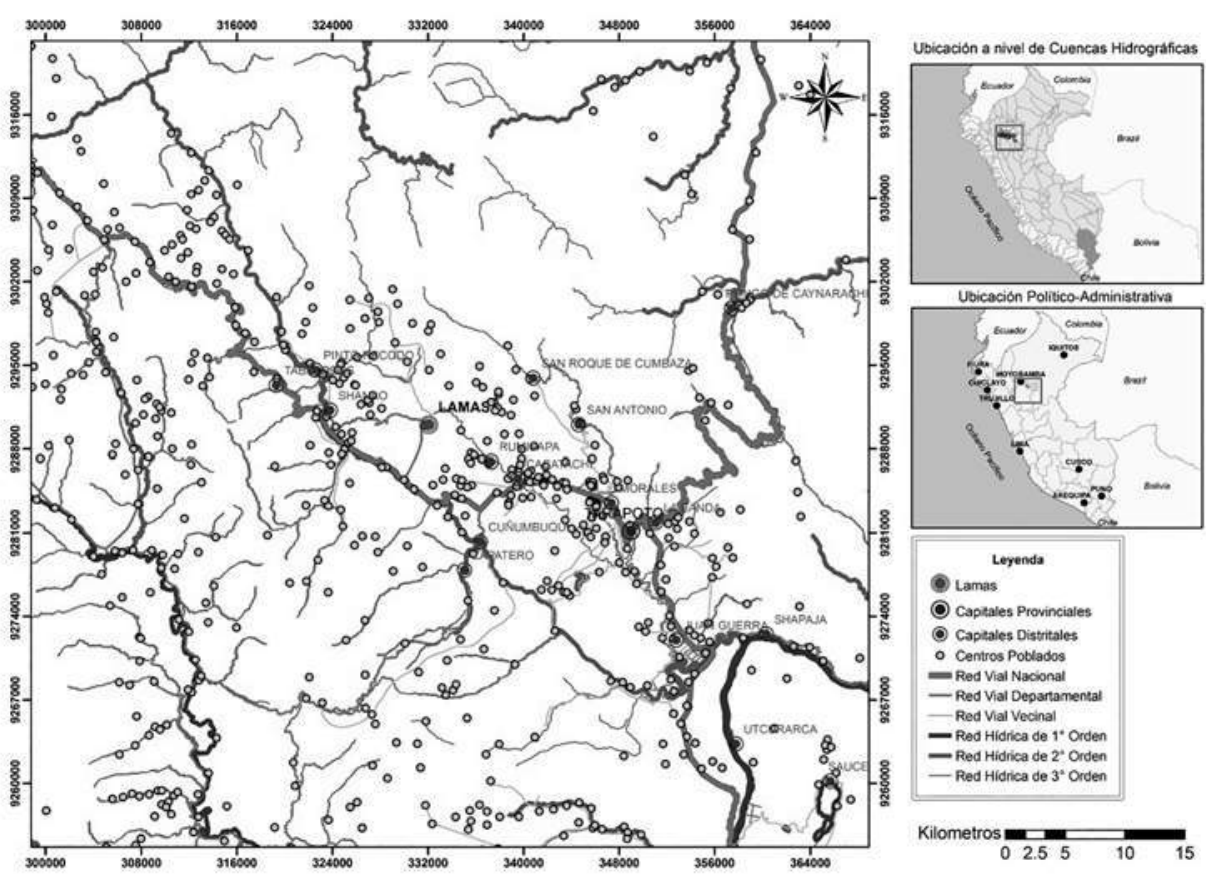

Figura 4. Mapa de Lamas y su contexto territorial. Fuentes: Instituto Geográfico Nacional (IGN), Ministerio de Transportes y Comunicaciones (MTC), Ministerio del Ambiente (Minam). Elaboración: Gustavo Rondón R.

La principal característica de la zona urbana de la ciudad de Lamas es la presencia de un barrio indígena, Wayku, que agrupa a la población "quechua-lamista», los cuales se encuentran en constante interacción con las zonas mestizas de la ciudad. Los «quechualamistas» son un grupo nativo que ha ocupado el territorio de Lamas desde la época pre-inca y se caracterizan principalmente por sus vestimentas (llenas de color) y la utilización de una variante local del quechua.

Al momento de la fundación española de la ciudad, los nativos fueron desplazados a la periferia de la ciudad (donde se encuentran actualmente), manteniendo sus tradiciones culturales y sus técnicas constructivas (Raimondi, 1929). A nivel hidrográfico, Lamas se encuentra ubicada en la cuenca baja del río Mayo, el cual nace en la región Amazonas y es un afluente del río Huallaga. A su vez, a nivel de subcuencas, la ciudad de Lamas forma parte de la red de ciudades que están comprendidas dentro de la subcuenca del Cumbaza (río que abastece de agua a Tarapoto). 


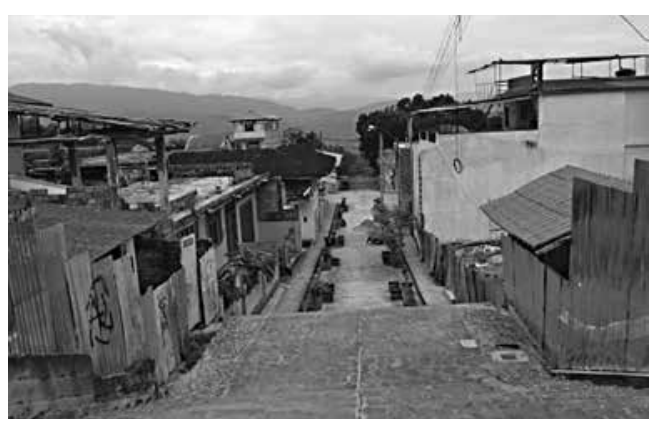

La ciudad se caracteriza por haberse adecuado al relieve del terreno. Por tal motivo es conocida como la "ciudad de los tres pisos».

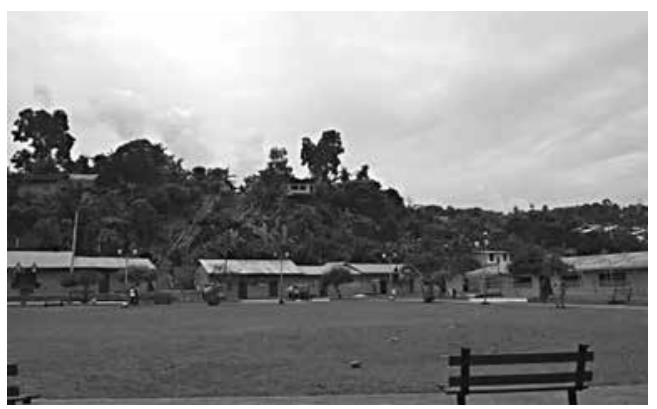

Otra particularidad de Lamas es la presencia de una población nativa, los quechua-lamistas, que se ubican en el barrio de Wayku. Ellos mantienen no solo tradiciones arquitectónicas (construcción en tapial) sino también en otras costumbres (vestidos, fiestas y formas de vida).

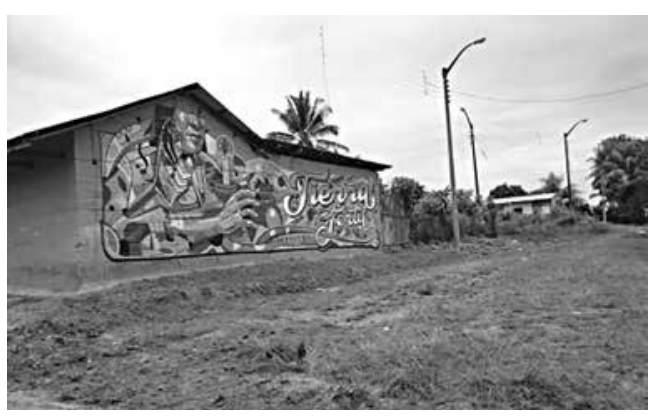

La importancia del hinterland para la ciudad de Lamas es muy alta. A pesar de verse influenciado por Tarapoto (en temas de servicios y turismo), Lamas sigue siendo un nodo y un centro de acopio para la producción agraria (principalmente café y cacao) de los alrededores. Es por eso que la tierra y el agua siguen siendo claves para el desarrollo de las actividades urbanas.

Figura 5. La ciudad de Lamas, su población y sus actividades. Fotos: Gustavo Rondón R.

Capital de la provincia de Condorcanqui (región Amazonas, la región más occidental de la Amazonía peruana) el pequeño pueblo de Santa María de Nieva tiene una población de 3329 habitantes (INEI, 2007). Este número resulta de la suma de cuatro pequeños centros poblados que con la expansión urbana se han consolidado como un dos continuos urbanos separados por el río Nieva (Santa María de Nieva, Juan Velasco Alvarado, La Tuna y Héctor Peas). A nivel distrital, la población es predominantemente rural (85\%): 18919 habitantes, mientras que la población urbana del distrito está concentrada en Nieva. A nivel de la provincia, donde también predomina la población rural (85\% de la población total), Nieva concentra a casi el $60 \%$ de la población urbana de la provincia. 


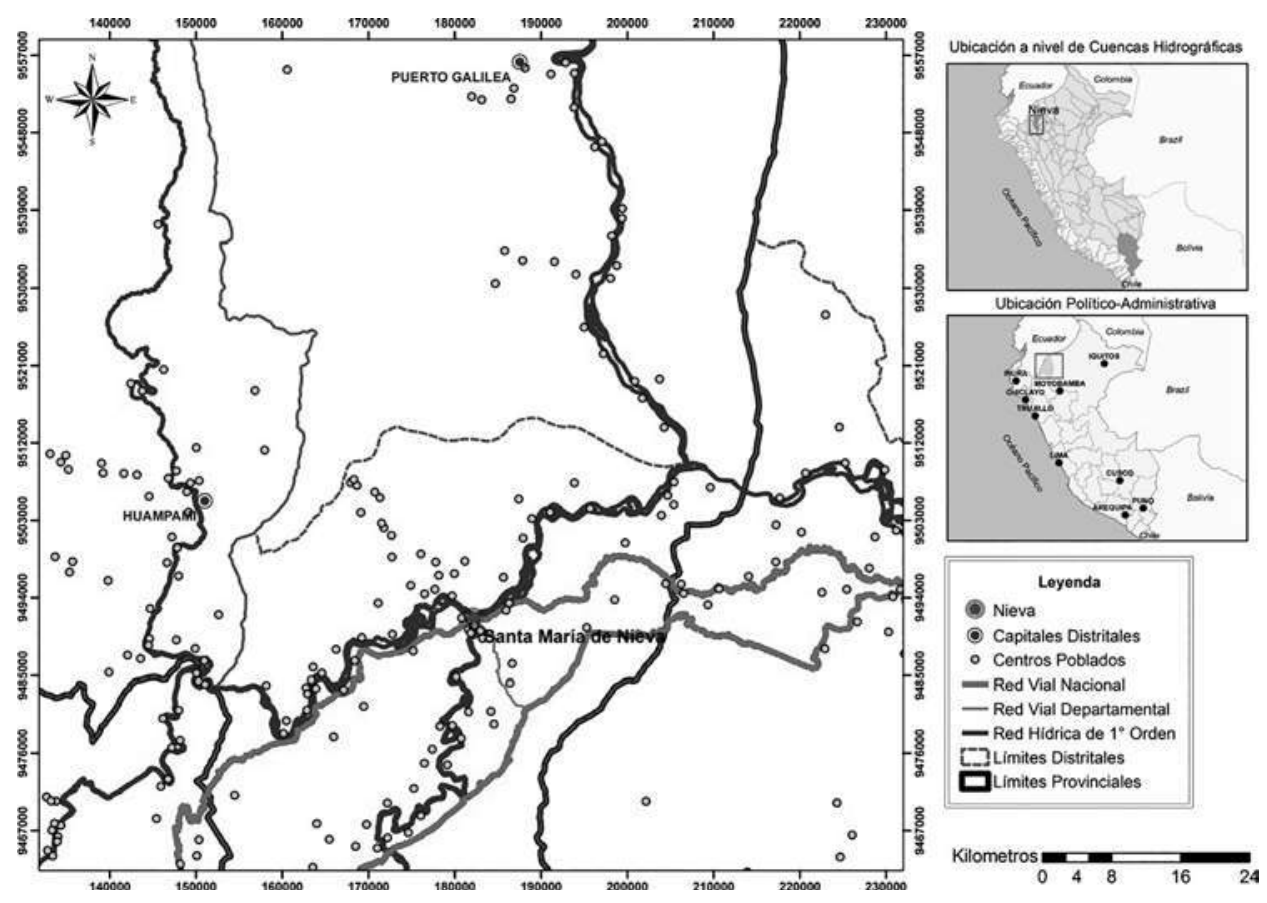

Figura 6. Mapa de Santa María de Nieva y su contexto territorial. Fuentes: Instituto Geográfico Nacional (IGN), Ministerio de Transportes y Comunicaciones (MTC), Ministerio del Ambiente (Minam). Elaboración: Gustavo Rondón R.

La actividad principal de la ciudad es el comercio, el cual depende en gran medida de las actividades agroforestales desarrolladas en su hinterland. Además, Santa María de Nieva es el punto de recolección de todos los productos agrícolas que serán vendidos en la costa y es el punto de acopio de los productos costeros o andinos que serán distribuidos en los centros poblados.

Además, la ciudad es el lugar donde las comunidades indígenas (awajún-wampis) acceden a la vida urbana y «occidental». Antes de las primeras misiones españolas en estos territorios, los awajún-wampis se instalaron en las partes altas de los ríos, por ser zonas de protección natural frente a los enemigos. Este modelo de ocupación fue modificada con la instalación de las misiones religiosas, el desarrollo de escuelas de las misiones y la explotación de los recursos naturales (Guallart, 1997). A nivel hidrográfico Santa María de Nieva forma parte de la cuenca del río Maranón, puesto se ubica en la desembocadura del río Nieva con el río Marañón, muy cerca del pongo de Manseriche, el cual es la entrada no solo a la región de Loreto, sino también es la transición entre la «selva alta» y la «selva baja». 

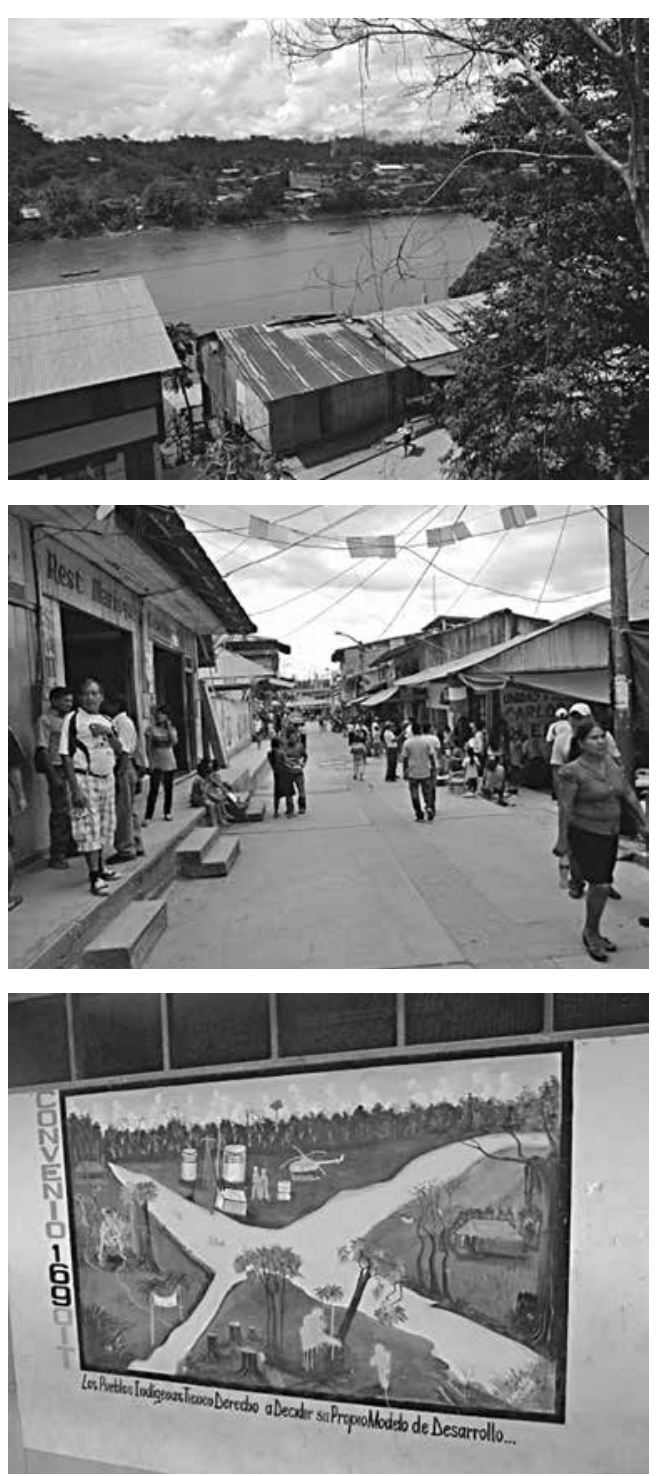

Santa María de Nieva se caracteriza por estar dividida en dos por el río Nieva: en la margen izquierda se ubican los barrios más antiguos de la ciudad (Plaza de Armas, Iglesia, etc.) y en la margen derecha están los nuevos barrios.

La actividad económica principal en Nieva es el comercio. Todos los productos que las comunidades nativas producen en sus "chacras», son vendidos/intercambiados en la zona urbana. El asfaltado de la carretera permite que los productos/servicios lleguen con más facilidad.

La relación entre la población y el territorio ha cambiado con el tiempo. El modelo de ocupación y uso de los recursos actual tiende a no respetar la armonía antes existente. Sin embargo, las comunidades nativas siempre muestran su deseo de lograr una sostenibilidad en el modelo de desarrollo.

Figura 7. La ciudad de Nieva, su población y sus actividades. Fotos: Gustavo Rondón R.

El estudio de dos ciudades amazónicas permitirá realizar un análisis comparativo ya que encontramos tanto similitudes como diferencias, las cuales serán expuestas a continuación. Lamas y Santa María de Nieva presentan una población indígena que ha ocupado el territorio antes de la llegada de los primeros colonos y migrantes. Como resultado, hoy en día podemos observar una diferencia entre las formas de uso y tenencia de la tierra y el uso de recursos naturales entre las poblaciones nativas con las nuevas 
colonias «occidentales» que se establecieron en estos territorios. Se puede señalar existía una «armonía» entre las comunidades indígenas y su territorio que comenzó a fracturarse o quebrarse con la introducción del modelo económico-productivo que consideraba al territorio y todos sus elementos solo como un recurso. Otra similitud entre las dos localidades de estudio es que las dos tienen la categoría político-administrativa de capital provincial. Este no es un detalle menor, porque como capitales provinciales, estas localidades concentran los poderes públicos creando una dependencia (obligada) sobre las poblaciones que habitan sus hinterlands debido a que se hace necesario ir hacía ellas para realizar procesos administrativos, legales o alguno relacionado con el poder estatal ahí instalado.

Sin embargo, no solo hay similitudes entre las dos localidades de estudio. En cuanto a la ubicación, Lamas se encuentra en una zona de relieve heterogéneo, con fuertes pendientes, en una zona alejada de los principales ríos (Mayo y Huallaga). Por el contrario, Santa María de Nieva se encuentra en una zona un poco más plana cerca de los principales ríos (Marańón y Nieva). Aunque ambas ciudades tienen una gran población indígena, existe un nivel de apropiación del espacio urbano diferente. En Lamas, es claro que existe un barrio indígena (una minoría a nivel de población) dentro de la ciudad, donde encontramos una población mayoritariamente nativa que vive y participa de la dinámica «occidental» de la ciudad, manteniendo eso sí, algunas prácticas culturales como la vestimenta, la tradición constructiva de las casas, la elaboración de artesanías, etc. En el caso de Santa María de Nieva, por el contrario y a pesar de que la población nativa es mayor en proporción a la migrante, en el espacio urbano se mimetiza con esta población mestiza y no muestra claramente su origen nativo en la mayoría de casos (siendo sus comunidades nativas de origen el lugar reservado para mostrar sus prácticas culturales).

Por último, otra diferencia entre las dos localidades de estudio es el papel que ha jugado (y lo continua haciendo) la carretera como generador de nuevas dinámicas territoriales. En el caso de Lamas, la construcción de la "Carretera Marginal de la Selva» (siglo XIX) ha llevado a una disminución dinámica económica, dejando a la ciudad de Tarapoto como polo de intercambio comercial entre Yurimaguas y las ciudades costeras. Esto ha provocado que Lamas, a pesar de ser un centro de poder administrativo por su rol de capital provincial, se haya vuelto dependiente de Tarapoto en términos de desarrollo económico (la mayor parte de la producción agraria del hinterland de Lamas — café y cacao — va directo a Tarapoto). La otra versión de la historia se refleja en Santa María de Nieva, que vive un proceso totalmente diferente. La carretera que recién en este año (2015) ha sido asfaltada totalmente viene generando nuevas dinámicas tanto como para el espacio urbano y los distintos centros poblados que se encuentran cerca del trazo, convirtiéndose en la forma más rápida de transportar los bienes y productos desde/hacia Nieva. 


\section{LA METODOlOGía: TÉCNICAS MIXTAS PARA AFRONTAR UNA REALIDAD COMPLEJA}

Teniendo en cuenta estas características de ocupación del territorio y a través de un levantamiento de información de campo, sumado al análisis de fuentes cartográficas (mapas e imágenes satelitales) se realizó un primer estudio del ciclo urbano del agua, basado en la idea de que este ciclo no solo se realiza de manera íntegra en el ámbito urbano y que evidentemente presenta una relación estrecha con el territorio (y sus recursos) de ambas localidades.

El primer paso consistió en identificar a nivel general cuáles eran los actores que conformaban el sistema de uso-manejo-gestión del agua, tanto en Lamas como en Santa María de Nieva. Para eso se utilizó un análisis de redes sociales, ya que, el análisis de redes sociales puede analizar por un lado las estructuras generales de una red (actores y sus características) y por el otro lado las interacciones o relaciones entre ellos (Schweers, 2002). Según Hoogendam (1999) las tareas a cumplir por esta red se dividen en cuatro principales campos de acción:

- El uso y manejo del agua

- Monitoreo y control

- Definición de políticas

- Inversión

La complejidad del abanico de actores tiene su origen en la cantidad de usos y características del recurso agua. Así, por ejemplo, es importante reconocer en primer lugar, y para ir entendiendo la cantidad de actores relacionados al recurso, que más allá de los límites políticos-administrativos y los roles institucionales, el agua es un recurso que traspasa lo geográfico, lo ecológico y lo social. Un mismo curso de agua puede atravesar diferentes jurisdicciones territoriales y políticas, diferentes pisos altitudinales y ser usado con diferentes propósitos por diferentes actores localizados en sitios espacialmente diferentes (FFLA, 2007).

Además, se tuvo que realizar una división entre los actores y los actores claves. Un actor es todo individuo, que se encuentra o forma parte de un grupo que tiene relación directa o indirecta con el proyecto a ejecutar. El actor clave es aquel individuo cuya participación es indispensable y obligada para el logro del propósito, objetivos y metas del proyecto en cuestión. Tienen el poder, la capacidad y los medios para decidir e influir en campos vitales que permitan o no el desarrollo del proyecto (Conagua, 2008).

La figura 8 resume de manera secuencial cuáles fueron los pasos que se siguieron para levantar la información acerca del ciclo urbano del agua en las dos localidades de estudio: 


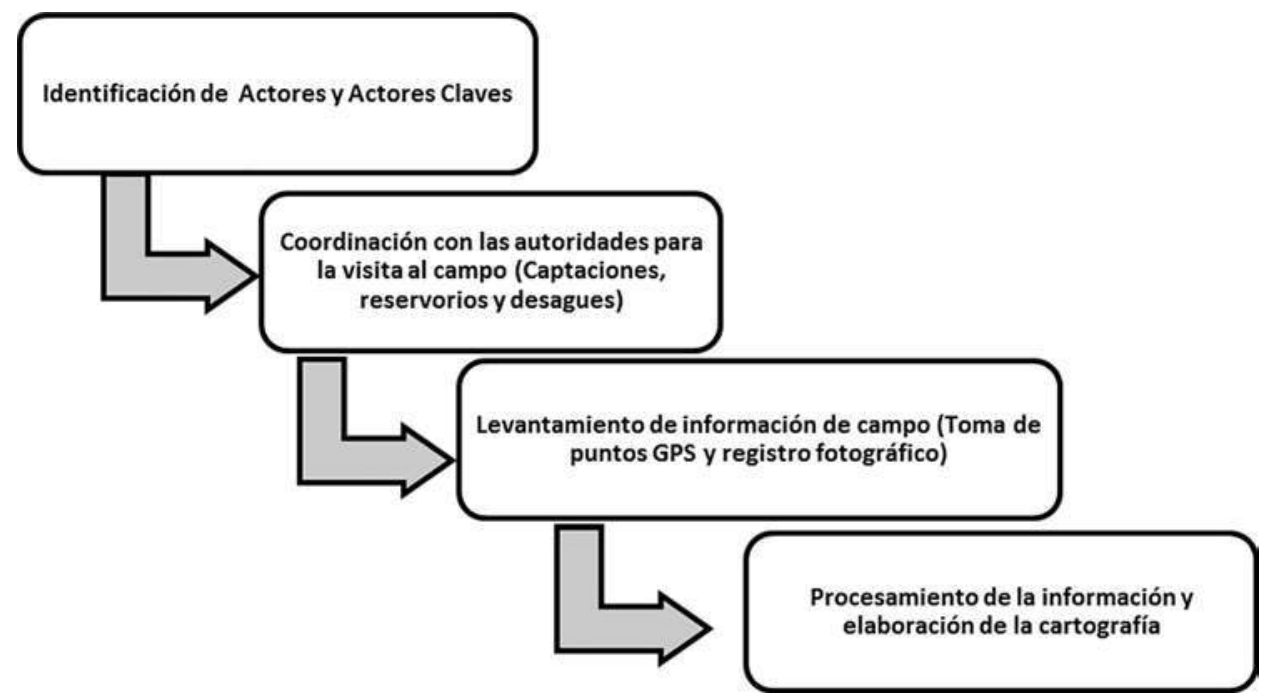

Figura 8. Proceso de levantamiento de información de campo.

Elaboración: Gustavo Rondón R.

Este primer acercamiento metodológico a la problemática de ciclo urbano del agua y sus impactos en el territorio se basa en una aproximación mixta pues se utilizan tanto técnicas cualitativas (como pueden ser entrevistas, observaciones de campo, conversaciones informales con los actores, autoridades, etc.), así como también técnicas cuantitativas (análisis de imágenes satelitales, fotos aéreas, datos censales, etc.). Como señala Gondard-Delcroix (2006), la confrontación entre el pragmatismo de los métodos cuantitativos y la ductilidad e intuición de los métodos cualitativos permite reconocer la complejidad de los fenómenos estudiados.

En nuestro caso de estudio, la complejidad que representa la gestión del agua en Lamas y Santa María de Nieva representa un reto para la aplicación de este enfoque metodológico. Además, como se verá en los resultados, en base a información cualitativa que ha sido recogida en entrevistas y charlas informales con los actores; sumado a la toma de puntos GPS y la elaboración de una cartografía local se ha generado una base de datos mixta, que combina tanto el saber local como el conocimiento científico, reflejados en un solo conjunto de información espacial.

\section{Resultados}

El trabajo de campo realizado durante el mes de agosto del presente año permitió identificar y conocer las fuentes de agua que abastecen a la ciudad de Lamas. La fuente principal es la quebrada Chontal y la segunda fuente es la quebrada Juanjuisillo (la cual tiene tres captaciones: una es la quebrada y también existen dos manantiales). 


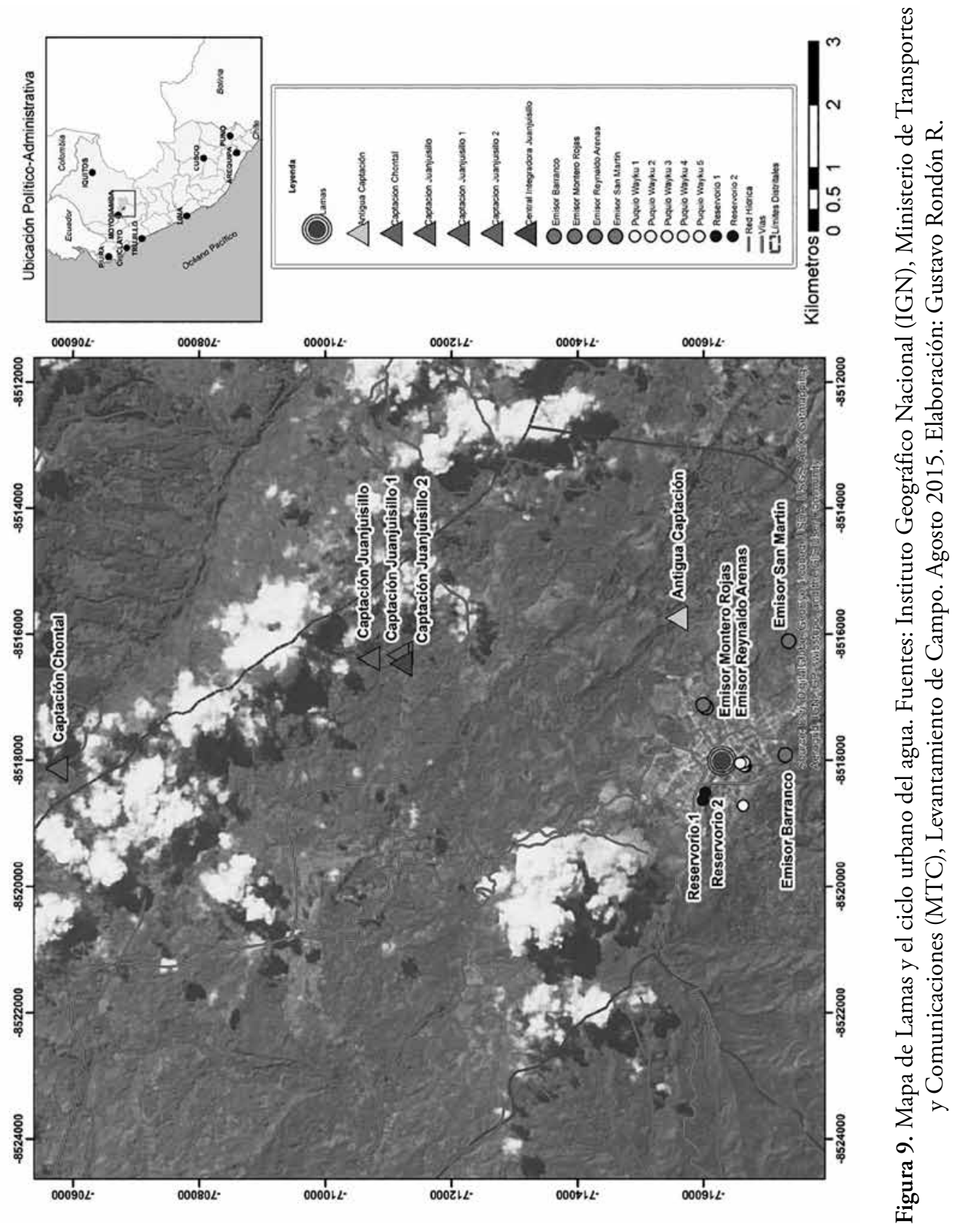




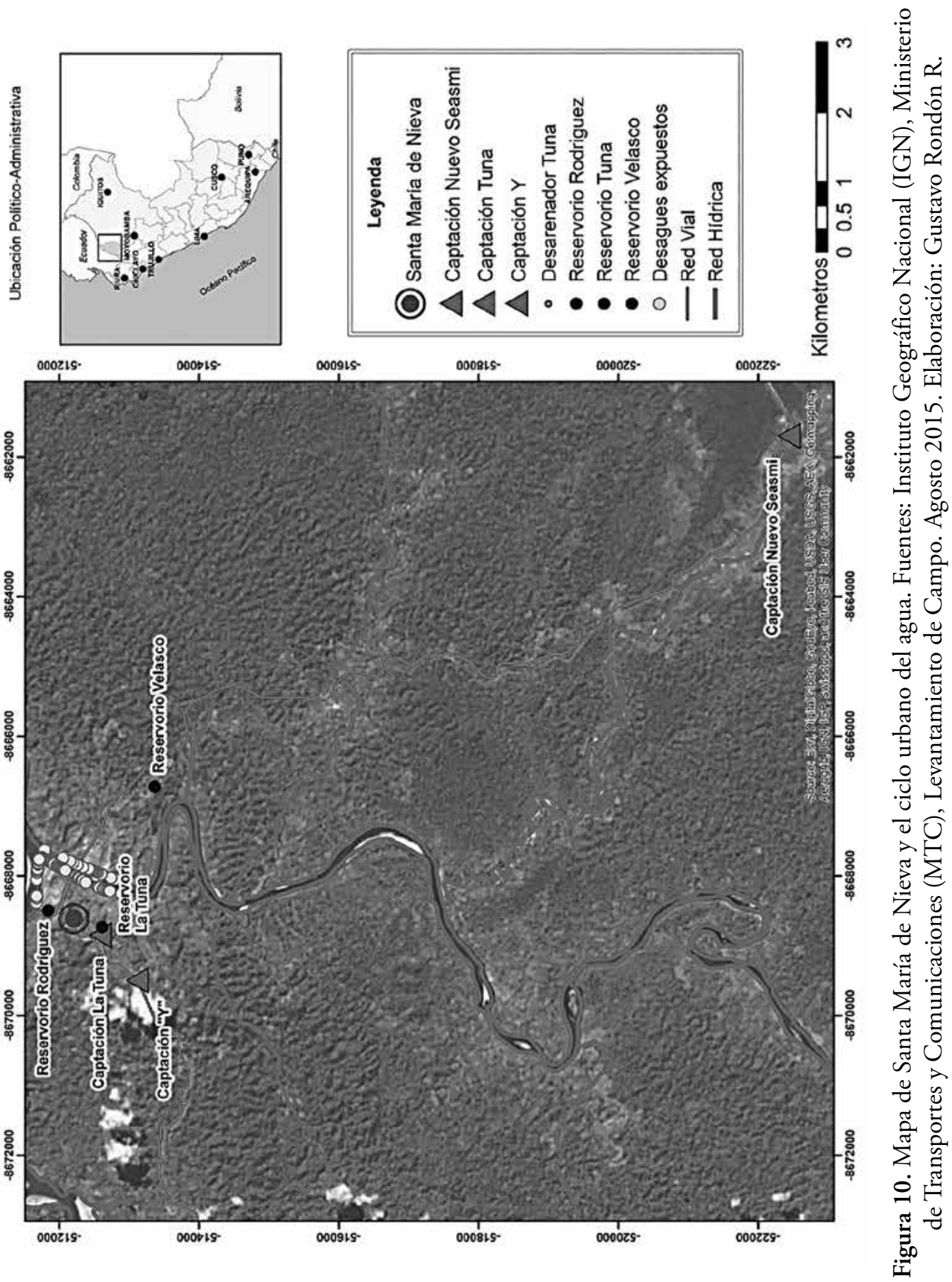




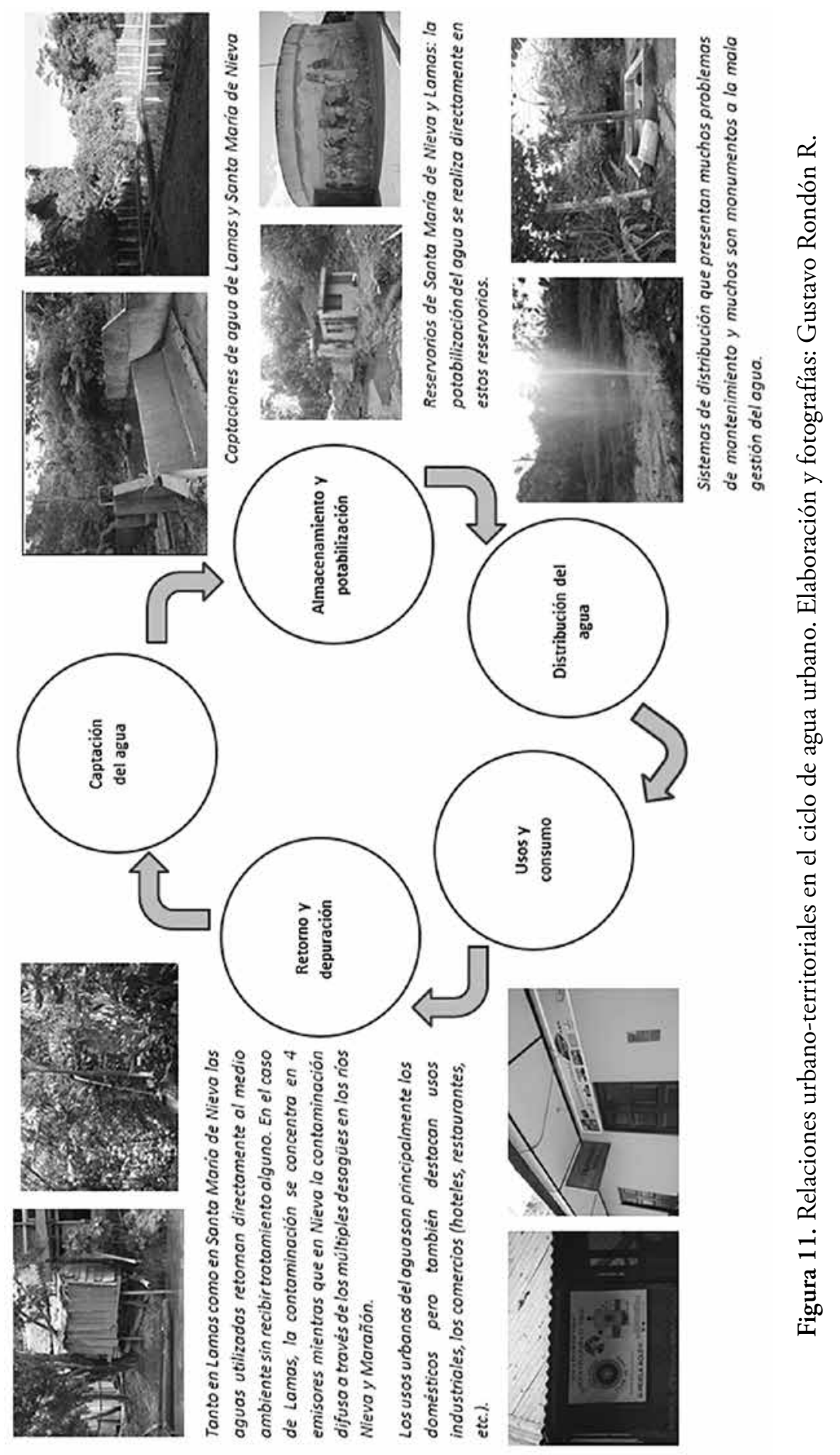


Estas captaciones se concentran en una central donde se juntan y se dirigen a los dos reservorios ubicados en la ciudad de Lamas. Es en los reservorios donde el agua que es captada de las quebradas y manantiales se potabiliza, tarea que recae en la Empresa Municipal de Agua Potable y Alcantarillado. El transporte del agua desde las zonas rurales a la ciudad de Lamas se realiza por gravedad pues las captaciones se encuentran a una mayor altitud que los reservorios.

Respecto a los llamados emisores, que en la práctica son los grandes colectores de desagüe, la ciudad de Lamas tiene cuatro emisores debido a la topografía de la ciudad. Cada emisor recoge los desagües de los distintos barrios y estos desechos son vertidos directamente a otras quebradas o pequeños riachuelos que claramente afectan a la calidad del medio ambiente.

En el caso de Santa María de Nieva, tal vez producto de su ubicación territorial, las captaciones de agua se encuentran tanto cerca de la zona urbana y también un poco más alejada. La captación principal se encuentra en el centro poblado Nuevo Seasmi, donde el agua de la quebrada del mismo nombre lleva el agua hasta Santa María de Nieva. Esta captación lleva el agua hasta el reservorio de Velasco para abastecer a las viviendas asentadas en la margen derecha del río Nieva. Además, a través de un punto aéreo esta misma captación abastece a algunos barrios de la zona del margen izquierdo. Otra captaciones que abastecen a la población de Santa María de Nieva son la de La Tuna, ubicada en la parte alta del barrio del mismo nombre y la captación conocida como la «Y».

Respecto a los desagües, el tema es crítico en Santa María de Nieva. A lo largo del Marañón y el Nieva se han georreferenciado más de 50 desagües que vierten las aguas usadas directamente a los ríos (esto sin contar todos los residuos sólidos). Un punto crítico resultan unos pozos ubicados en las orillas del río Marañón que debieron ser pozos de tratamiento del agua pero por malos manejos solo han quedado como depósitos de aguas servidas.

\section{DISCUSIÓN Y REFLEXIONES}

Los resultados obtenidos muestran que, al nivel de las fuentes de agua y del retorno de las aguas utilizadas, ambas localidades tienen un gran impacto en el territorio; además, se puede apreciar que el agua es un elemento determinante en la configuración o delimitación de subzonas dentro del espacio urbano.

El ciclo urbano del agua, como se mostró en la figura 11, no solo tiene un impacto en el ámbito urbano. Las captaciones de agua, tanto en Santa María de Nieva como en Lamas se ubican en zonas rurales alejadas de la ciudad y también en espacios periurbanos. En estas captaciones, no solo es recurso agua es manejado por las autoridades competentes (Emapa en el caso de Lamas y la misma Municipalidad Provincial 
de Condorcanqui en el caso de Nieva) sino que también el terreno dónde se ubican estas captaciones ha tenido que ser intervenido.

En el caso de Nieva la municipalidad compró los terrenos donde se ubican las captaciones de agua y ha realizado un cerco perimétrico para evitar la entrada de personas. En el caso de Lamas, Emapa no ha podido comprar los terrenos donde se encuentran las captaciones pues estos terrenos pertenecen a una comunidad campesina que aún no tiene título de propiedad, por lo tanto, solo han podido «alquilar» el terreno para poder así darle un adecuado cuidado. Estos dos casos demuestran de manera clara que si bien el ciclo urbano del agua se inicia con la captación del recurso, el agua generalmente no se encuentra en la zona urbana por lo que se genera un vínculo urbano-territorial respecto a la gestión del recurso.

Como señalan Canziani y Schejtman (2013), la preocupación por los problemas del desarrollo urbano no pueda abordarse sino a partir de su relación con los espacios rurales de los territorios que comparte. Y esta preocupación se refleja en el esfuerzo (dentro de sus capacidades y posibilidades) de las autoridades encargadas del tema de abastecimiento de agua por cuidar el terreno de las captaciones: evitan la deforestación de las zonas cercanas a las quebradas, realizan reforestación con árboles variados y se preocupan por mantener limpias las captaciones.

Respecto a la disposición final del agua utilizada en este ciclo urbano del agua, ambas ciudades están afectando negativamente al medio ambiente, especial a la calidad de las fuentes de agua. Tanto Lamas como Nieva no disponen de un sistema de tratamiento de aguas usadas, todas van directamente a quebradas, barrancos (en el caso de Lamas) y ríos (en el caso de Nieva). Si bien el tamaño de las dos zonas urbanas no puede ser catalogado como grande o mediano, es innegable el impacto negativo sobre la calidad del agua. En Lamas la contaminación se concentra en cuatro colectores de los desagües urbanos, mientras que en Nieva el impacto es mucho más visual pues uno puede observar más de 50 desagües expuestos a lo largo de las orillas del río Nieva y del río Marañón. Respecto al mismo tema es curioso señalar que en ambas localidades hubo proyectos para realizar plantas de tratamiento de aguas, los cuales terminaron siendo otros grandes monumentos a la mala gestión del agua pues solo se instaló la infraestructura más nunca funcionaron.

Lo observado y analizado en el trabajo de campo, sumado a la afirmación de Harvey (1996), quien señaló que no hay nada a priori no natural en una represa o en un sistema de riego, Swyngedouw (2004) muestra que los procesos de circulación (flujo) del agua ponen en evidencia los procesos políticos, económicos, sociales y ecológicos a diferentes escalas. La circulación (flujo) del agua es un proceso social y físico combinado, un flujo socionatural híbrido que fusiona Naturaleza y Sociedad inseparable (Larsimont y Grosso, 2014). Por otra parte, la idea de movimiento nos invita a entender cómo los flujos de agua, de capital y de poder están vinculados físicamente (Swyngedouw, 2004). 
Este ciclo hidrosocial del agua se refleja tanto en los distintos usos del agua en las localidades de estudio, así como en la relación que pueden tener las comunidades nativas (los quechua-lamistas y los awajún-wampis) con el agua que llega a ser muy distinta a la relación que tienen los habitantes urbanos.

Finalmente, el título hace referencia a los territorios del agua. Este concepto intenta reflejar en primer lugar el contexto territorial en que se encuentran las localidades de estudio: la Amazonía del Perú, la cual tiene la mayor disponibilidad física de agua que muchas veces no se refleja en la disponibilidad (cantidad y calidad) real de agua para la población y sus activades. Además, intenta ser un poco el reflejo de un concepto utilizado por distintos autores en los últimos años: «paisajes del agua». Los autores consultados en la literatura han destacado tres dimensiones que engloban el concepto: Latour $(1997,2004)$ Swyngedouw (1999) y Blanchon (2009) mencionan que el paisaje del agua refleja la naturaleza híbrida de los paisajes del agua y por lo tanto se presenta como un intento de cuestionar el cisma ontológico entre la sociedad y naturaleza; otros autores como Molle (2012) y Massey (2009) el paisaje del agua es una unidad de paisaje analizada a través de la lente donde de un lado tenemos los recursos hídricos, y en el otro lado, tenemos la interconectividad entre lo hidrológico, sociopolítico y el ecosistema; en otras palabras, representa el medio en el que se llevan a cabo los procesos del ciclo hidrosocial del agua.

El concepto de paisaje del agua intenta ser espacializado o territorializado para el contexto de las localidades de estudio. Así como en la introducción hablamos de las múltiples amazonias, en el caso del agua también podemos hablar de no solo un territorio del agua, sino de varios. La presente investigación es solo el inicio de este largo camino para descubrir y comprender cuáles son estos territorios del agua en Lamas y Santa María de Nieva. Al ser el agua un elemento transversal a todo el territorio, sus dominios pueden ser físicos (cuencas hidrográficas), político-administrativos (límites distritales o provinciales), económico-productivos (usos del agua) y socioculturales (tradiciones ancestrales en el manejo del agua, delimitación de barrios dentro de la ciudad según el uso del agua, etc.).

\section{Bibliografía}

Aguilera, F. 2006. Hacia una nueva economía del agua: cuestiones fundamentales. En: Polis, hacia una cultura del agua. Puesto en línea el 08 agosto 2012, consultado el 25 abril 2013. URL: http://polis.revues.org/5044

Agurto Belloso, J. (2006). El agua desde la visión andina, Condesan-InfoAndina. Nodo Regional para América Latina del Mountain Forum. Lima.

Autoridad Nacional del Agua (ANA) (2013). Plan Nacional de Recursos Hidricos del Perú. Lima, Perú. En proceso de aprobación. 
Bernex, N., Montes, L. (1992). Una aproximación a la lectura del espacio: cuenca del río Sisa-Huallaga Central y Bajo Mayo. Lima: PUCP-CIGA/PEHCBM

Blanchon, D. (2009). L'espace hydraulique sud-africain: Le partage des eaux. París: Karthala.

Budds, J. (2012). La demanda, evaluación y asignación del agua en el contexto de escasez: un análisis del ciclo hidrosocial del valle del río La Ligua, Chile. Revista de Geografía Norte Grande, 52, 167-184. Chile. http://dx.doi.org/10.4067/S0718-34022012000200010

Cabrera, J. (2014). Fragmentation urbaine à travers des réseaux L'exemple de stratégies locales de gestion de l'eau dans la municipalité de Quillacollo du département de Cochabamba, Bolivie. Tesis doctoral. Université de Liege.

Canziani, J. \& Schejtman, A. (2013). Ciudades intermedias y desarrollo territorial. Lima: Fondo Editorial de la Pontificia Universidad Católica del Perú. RISMIP Centro Latinoamericano para el Desarrollo Rural.

Comisión Nacional del Agua (Conagua) (2008). Guía de Identificación de actores clave. Componente: Planeación Local, Proyectos Emblemáticos. Serie: Planeación Hidráulica en México.

De la Torre, A. (2012). Fundamentos para el Plan Nacional de Recursos Hidricos. Lima: Sociedad Geográfica de Lima, Perú.

Fundación Futuro Latinoamericano (FFLA) (2007). Diagnóstico y mapeo de actores, relaciones y conflictividad de la hoya de Quito. Quito, octubre.

Gondard-Delcroix, C. (2006). La combinaison des analyses qualitative et quantitative pour une étude des dynamiques de pauvreté en milieu rural malgache. Thèse pour le Doctorat en Sciences Économiques. Université Montesquieu Bordeaux IV.

GRADE-IEP (2014). Amazonía peruana y desarrollo económico. Editores: Roxana Barrantes y Manuel Glave. Lima, Perú.

Guallart, J. M. (1997). La tierra de los cinco ríos. Lima: PUCP: Banco Central de Reserva del Perú.

Harvey, David (1996). Justice, Nature and the Geography of Difference. Cambridge: Blackwell.

Hoogendam, P. (1999). Aguas y municipios, Retos para la gestión municipal de agua. Cochabamba: PEIRAV, Plural Editores.

Instituto Nacional de Estadistica e Información (INEI). 2007. Censos Nacionales: XI de Población y VI de Vivienda.

Larsimont, R. \& Grosso, V. (2014). Aproximación a los nuevos conceptos híbridos para abordar las problemáticas hídricas. Cardinalis. Revista del Departamento de Geografía, 2(2), $1^{\circ}$ semestre, $27-48$.

Latour, B. (1997). Nous n'avons jamais été moderne». En Essai d'anthropologie symétrique. Paris: La Découverte.

Latour, B. (2004). Politiques de la nature: Comment faire entrer les sciences en démocratie. París: La Découverte. 
Linton, J. (2010). What is Water? The History of a Modern Abstraction. Vancouver: UBC Press.

Massey, D. (2009). Concepts of space and power in theory and in political practice. Doc. Anàl. Geogr. 55, 15-26. España.

Molle, F. (2012). La gestion de l'eau et les apports d'une approche par la political ecology. En Gautier, D. \& Benjaminsen A.T. (Dirs.), L'approche Political Ecology: Pouvoir, savoir, environnement (pp. 219-240). París: Quae.

Pereyra, C. (2005). Panorama de los conflictos por el agua en el Perú. En Seminario Conflictos sociales, acceso al agua y previsiones estratégicas en el Perú. Lima: Iproga.

Pulgar Vidal, J. (1982). Le tre selve dell’ Antisuyu. En G. Majani Ronzoni (Ed.), T. Baldi Guarinoni (Trad.), Antisuyu ultimo sogno inca (pp. 109-122). Venecia: Erizzo Editrice.

Pulgar Vidal, J. (2014). Geografía del Perú. Las ocho regiones naturales. Editado por Nicole Bernex. Lima: Pontificia Universidad del Perú, Instituto de Ciencias de la Naturaleza, Territorio y Energías Renovables.

Raimondi, A. (1929). Almanaque peruano y guía de forasteros. En El Perú, tomo III, p. 42.

Schweers, C. (2002). Theoretische Grundlagen der Netzwerkanalyse. http://www.anubaonline.de/extdoc/Materialien_der_BNW_Fortbildung/BNW_bewerten/4-1-2Netzwerkanalyse.pdf

Swyngedouw, E. (1999). Modernity and Hybridity: Regeneracionismo, the Production of Nature and the Spanish Waterscape, 1890-1930. Annals of the Association of American Geographers 89(3), 443-465. http://dx.doi.org/10.1111/0004-5608.00157

Swyngedouw, E. (2004). Social Power and the Urbanization of Water: Flows of Power. Oxford: Oxford University Press.

Swyngedouw, E. (2009). The Political Economy and Political Ecology of the Hydro-Social Cycle. Journal of Contemporary Water Research \& Education, 142, 56-60. http://dx.doi. org/10.1111/j.1936-704X.2009.00054.x

Fecha de envío: 14 octubre, 2015 Fecha de aceptación: 25 noviembre, 2015 